\title{
Juvenile Idiopathic Arthritis
}

National Cancer Institute

\section{Source}

National Cancer Institute. Juvenile Idiopathic Arthritis. NCI Thesaurus. Code C114357.

A group of chronic, inflammatory childhood disorders of unknown etiology that primarily involve joints. 